\title{
The Epidemiologic Transition of Diabetes Mellitus in Taiwan: Implications for Reversal of Female Preponderance from a National Cohort
}

\author{
Chin-Hsiao Tseng ${ }^{*}, 1,2,3$
}

\author{
${ }^{I}$ National Taiwan University College of Medicine, Taipei, Taiwan \\ ${ }^{2}$ Division of Endocrinology and Metabolism, Department of Internal Medicine, National Taiwan University Hospital, \\ Taipei, Taiwan \\ ${ }^{3}$ Department of Medical Research and Development, National Taiwan University Hospital Yun-Lin Branch, Yun-Lin, \\ Taiwan
}

\begin{abstract}
Diabetes prevalence studies show a female preponderance, especially in developed countries. This review summarizes the epidemiologic evidence for a possible transition in sexual preponderance in Taiwan. Major epidemiologic surveys of diabetes prevalence and studies on the incidence and mortality of diabetes in Taiwan over the past decades were reviewed. Diabetes prevalence increased from 5.1\% in 1970 to $12.8 \%$ in 1996 with female preponderance. However, a 3-fold higher prevalence in men in the younger age of 19-44 years in contrast to a female preponderance in the older age was observed in the 1996 survey. The female preponderance could possibly be explained by a higher mortality due to higher prevalence of macrovascular complications and higher incidence of hypertension in the diabetic men. Although overall incidence of diabetes was higher in women (218.4 vs 187.1 per 100,000), age-specific incidence analyses suggested a higher incidence accompanied by obesity in men in the age groups below 45 years. In conclusions, increasing prevalence of diabetes with female preponderance had been observed for decades in Taiwan, which can be explained partly by the higher risk of morbidity and mortality in the diabetic men and an overall higher incidence in women of all ages. However, the sex preponderance in diabetes is expected to change from women to men rolling from the past decades to the future in Taiwan because of the increasing prevalence of obesity in the younger men.
\end{abstract}

Keywords: Diabetes mellitus, epidemiology, sexual preponderance, secular trend, Taiwan.

\section{INTRODUCTION}

Diabetes mellitus has become an epidemic disease with the highest morbidity and mortality around the world [1-3]. It is estimated that the case number has reached 171 million worldwide in 2000 and will rise to 366 million in 2030 [1]. The global burden of mortality attributable to diabetes was estimated to be 2.9 million, or $5.2 \%$ of all deaths [3]. According to an estimation of the global burden of diabetes, there are more women than men with diabetes, especially in developed countries [1].

Taiwan is a country with a population of approximately 22.6 million. It is one of the most crowded countries in the world, with a population density of 625 persons $/ \mathrm{km}^{2}$ in 2003 . Most of the people living in Taiwan are migrants or descendants of migrants from Mainland China; and approximately $2 \%$ of the population is indigenous people, probably of Malayo-Polynesian origin. According to a series of populationbased surveys, the prevalence of diabetes in Taiwan has been increasing with female preponderance over the past decades $[4,5]$. Theoretically, prevalence is a function of incidence and mortality. It was not known whether the increasing prevalence was resulted from changes of one or both of these

*Address correspondence to this author at the Department of Internal Medicine, National Taiwan University Hospital, No. 7 Chung-Shan South Road, Taipei, Taiwan; Tel/Fax: +886-2-2388-3578; E-mail: ccktsh@ms6.hinet.net two factors. A close observation of the secular trends in the epidemiologic transition of such a chronic and health damaging disease is important for both the policymakers and clinical practitioners to take early measures in the prevention and treatment of the disease.

To answer a series of these interrelated epidemiologic questions, it would be better if all data could be obtained from a large cohort of diabetic patients representative at a national level. The National Health Insurance (NHI) program in Taiwan is a unique and compulsory health care system which covers more than $96 \%$ of the total population. Only those who are serving in the military or subject to criminal sanction, etc. would be exempt from this program. A systematic use of the data obtained from a cohort of diabetic patients within this health care system has been conducted since the implementation of the NHI in 1995. Therefore it is the purpose of this review to give a general description of the data and interpretation of the implications introduced by a series of epidemiologic analyses from the established NHI diabetes cohort along with the vital statistics and the series of prevalence surveys in this country over the past decades.

\section{INCREASING PREVALENCE OF DIABETES}

In parallel to the economic growth indicated by the per capita gross national product and the per capita national income, the prevalence of diabetes has been increasing over the past decades in Taiwan (Fig. 1). The crude prevalence 


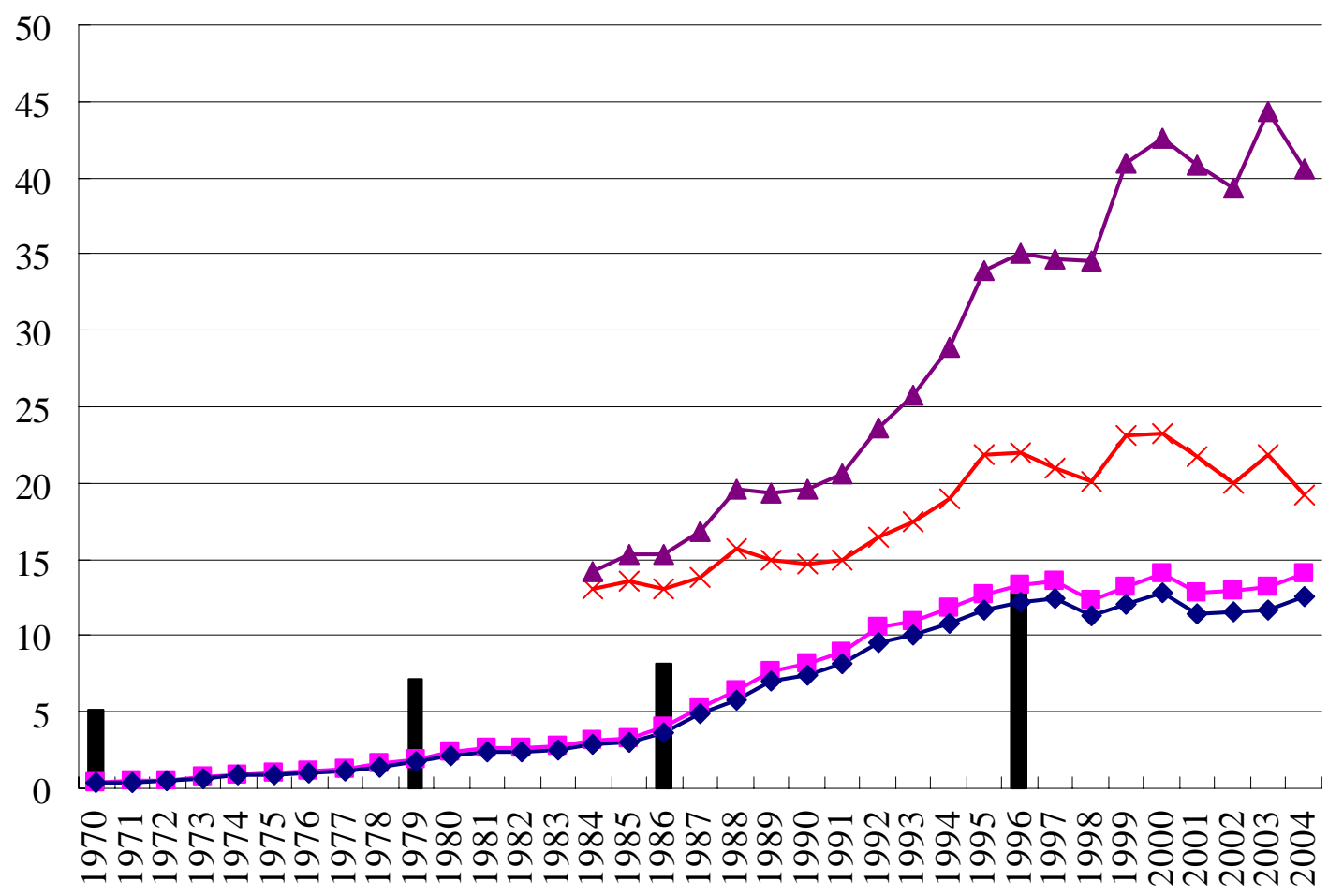

Year

Fig. (1). The prevalence of diabetes (black vertical columns, \%), crude mortality rate from diabetes (triangle, per 100,000 population), standardized mortality rate from diabetes (cross, per 100,000 population, standardized to the mid-year population of Taiwan in the year 1981), the per capita gross national product (square, in 1,000 US\$) and the per capita national income (diamond, in 1,000 US\$) in Taiwan over the past decades.

rates of diabetes in Taipei city for those aged $\geq 40$ years were $5.1 \%$ in $1970,7.1 \%$ in 1979 and $8.2 \%$ in 1986 , respectively (Fig. 1) [4]. The prevalence increased dramatically to $12.8 \%$ in a later survey in 1996 (Fig. 1) [5]. In consistent to the global estimate [1] the prevalence of diabetes was higher in women than in men in either the urban or rural area in the 1986 survey [4], and in the 1996 survey [5] (Fig. 2). It was interesting to notice a 3 -fold higher prevalence in men than in women in the younger age group of 19-44 years in contrast to a female preponderance in the older age groups in the 1996 survey (Fig. 2). The estimates in the younger age group might not be steady because diabetes is age-related and the number of diabetic patients in the younger age was small. However, if this was not spurious, an implication of a reverse trend in sex preponderance is expected in the years to come. This was evidenced by a recent national survey in more than 6,500 residents in 2002 showing a higher prevalence of diabetes in the male population aged below 60 years and a female preponderance in the older population (unpublished data).

\section{ESTABLISHMENT OF A DIABETIC COHORT}

To answer a series of epidemiologic questions on diabetes in Taiwan, a national cohort of 256,036 diabetic patients (cohort I) was recruited from 66 clinical settings claiming for the National Health Insurance and distributing evenly throughout Taiwan during the year 1995 to 1998 [6]. Among them 93,484 cases (cohort II) were successfully interviewed

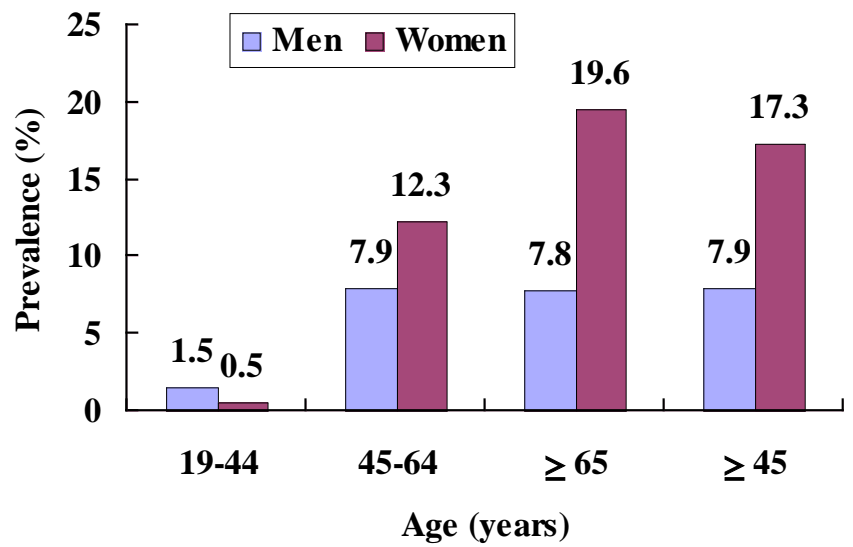

Fig. (2). Age- and sex-specific prevalence of diabetes in the Nutrition and Health Survey in Taiwan during 1993-1996 (figures adapted from reference No. [5]).

by telephone [7-13]. A further subsample of 16,994 cases (cohort III) was used for prospective long-term follow-up of diabetes-related complications [14]. Cohort IV was established from a random sample of cohort II with the collection of detailed personal information, biospecimens of urine and blood and complete laboratory examinations for diabetic chronic complications. A prospective follow-up study on the mortality of the diabetic patients was based on analysis of cohort I [6]. The incidence trend of diabetes [7], the prevalence of lower extremity amputation [8] and the sex 
difference in the incidence of hypertension [10] were based on analyses obtained from cohort II; and the prevalence of stroke in the diabetic patients [14] was based on cohort III. The following discussion will be based primarily on the data obtained from the above cohorts.

\section{TREND OF MORTALITY FROM DIABETES IN TAIWAN}

In Taiwan, the Ministry of Interior published the vital statistics of the country each year. The trend of mortality from diabetes according to these vital statistics has shown an increasing proportion of the national deaths ascribed to diabetes (Fig. 1). In 1960, 1970, 1980, 1990 and 2000 the proportion of mortality ascribed to diabetes in the population was $0.27 \%, 0.71 \%, 1.67 \%, 3.80 \%$ and $7.59 \%$, respectively; and diabetes respectively ranked as the $29^{\text {th }}, 18^{\text {th }}, 13^{\text {th }}, 5^{\text {th }}$ and $5^{\text {th }}$ cause of death. Mortality from diabetes in Taiwan swiftly jumped within the ten leading causes of death after the 1980s following the rapid economic growth of the country and was ranked as the fifth from 1987 to 2001 and as the fourth since 2002.

A longitudinal follow-up of 256,036 diagnosed diabetic patients recruited from 1995 to 1998 across Taiwan (cohort I) and followed up to the end of 2001 with a total of $1,124,348.4$ person-years has shown that the crude mortality rate was 39.0/1,000 person-years and the overall standardized mortality ratio was $1.63(1.62-1.65)$ [6]. Mortality rates increased with age in either sex (Fig. 3A). However, the diabetic men had a significantly higher mortality rate than women in all age strata (Fig. 3A). The mortality rate ratios between the diabetic men and women (figures on top of Fig. (3A)) and the standardized mortality ratios between the diabetic patients and the general population (Fig. 3B) became greater as age declined. Therefore, the diabetic women suffer from a lower mortality than their male counterparts in all age groups, and the younger diabetic patients would tend to have a higher risk of loosing their life than the older patients while compared to their age-matched general population. Because this is a prospective follow-up study with a large sample size and the verification of vital status by using the national death registry data in the study is complete as it is required as part of the household registration in Taiwan, the results are less affected by potential bias or confounders and are believed to be reliable. The results strongly suggest that the diabetic men more rapidly flow out of the prevalence pool than the diabetic women because of their higher mortality. This could partly contribute to the female preponderance as observed in diabetes prevalence studies.

\section{INCREASING TRENDS OF DIABETES INCIDENCE VARY WITH AGE AND SEX}

To obtain an incidence always implies a requirement of prospective follow-up of a cohort without diabetes for years to come. This is time-consuming and laborious and tends to suffer from a biased estimate if the follow-up rate or the number of incident cases is too low. It is even more difficult to obtain a series of incidence rates if we intend to explain the increasing trend of prevalence by the trend of incidence. Although there is probably little doubt regarding the increasing prevalence of diabetes, there was still a lack of data with regards to an increase in diabetes incidence in Taiwan until our recent analyses of the database obtained from cohort II, which demonstrated an increasing trend of diabetes incidence over a 5-year period from 1992 to 1996 [7]. As a whole, a 1.8-fold increase of incidence was observed for both sexes within these 5 years. The overall incidence of diabetes in men and women was 187.1 and 218.4 per 100,000 population, respectively. This seems to be in agreement with the prevalence surveys published previously showing female preponderance. However, when the agespecific incidences were calculated, a different scenario was noted. The female preponderance was only observed in the older ages of 45 years or more, in contrary to the male preponderance in the younger age groups (Fig. 4).

\section{SEX DIFFERENCE IN MORBIDITY IN DIABETIC PATIENTS}

Macrovascular complications are responsible for the majority of death causes in the diabetic patients [6]. Hypertension is a well recognized risk factor for the development of atherosclerosis and therefore is highly correlated with the macrovascular complications of diabetes. Since the diabetic men have a higher mortality rate than the diabetic women in Taiwan, it will be interesting to know whether a similar sex difference in the macrovascular complications or their common risk factor of hypertension can be observed. By analyzing the data obtained from cohort II, it was seen that the diabetic men in Taiwan had a significantly higher risk of developing hypertension than the diabetic women, with a relative risk of 1.09 (1.05-1.14) [10]. The prevalence rates of macrovascular complications including lower extremity amputation (cohort II) [8], stroke (cohort III) [14] and coronary heart disease (cohort III, unpublished data) were all higher in the diabetic men than in the women. Therefore, the higher mortality in the diabetic men than women (Fig. 3) might reflect a higher risk of diabetic macrovascular complications and their common risk factor of hypertension in the diabetic men than women.

\section{DISCUSSION}

The prevalence of diabetes mellitus has been increasing with female preponderance in previously published surveys in Taiwan. Whether the mortality and incidence of diabetes can be responsible for these epidemiologic phenomena are interesting and important. Furthermore, macrovascular disease, for which hypertension is an important risk factor, always accounts for the mortality in the diabetic patients. An elucidation of the sex difference in the risk of developing hypertension and macrovascular complications can directly explain the sex difference in mortality and prevalence of diabetes.

The prospective follow-up for the mortality of cohort I has shown that the diabetic men consistently showed a higher mortality rate than women in all age groups in Taiwan. This study gives part of the explanation to the female preponderance in diabetes prevalence. The incidence trends of type 2 diabetes from 1992 to 1996 for the different age groups in men and women calculated from cohort II suggest a possibility of reversal trend of sex preponderance in diabetes prevalence in the following years to come. This was evidenced from the recent national survey in 2002 showing male preponderance in diabetes prevalence in the population aged below 60 years in contrast to a female preponderance in the older population (unpublished data). 
(A)

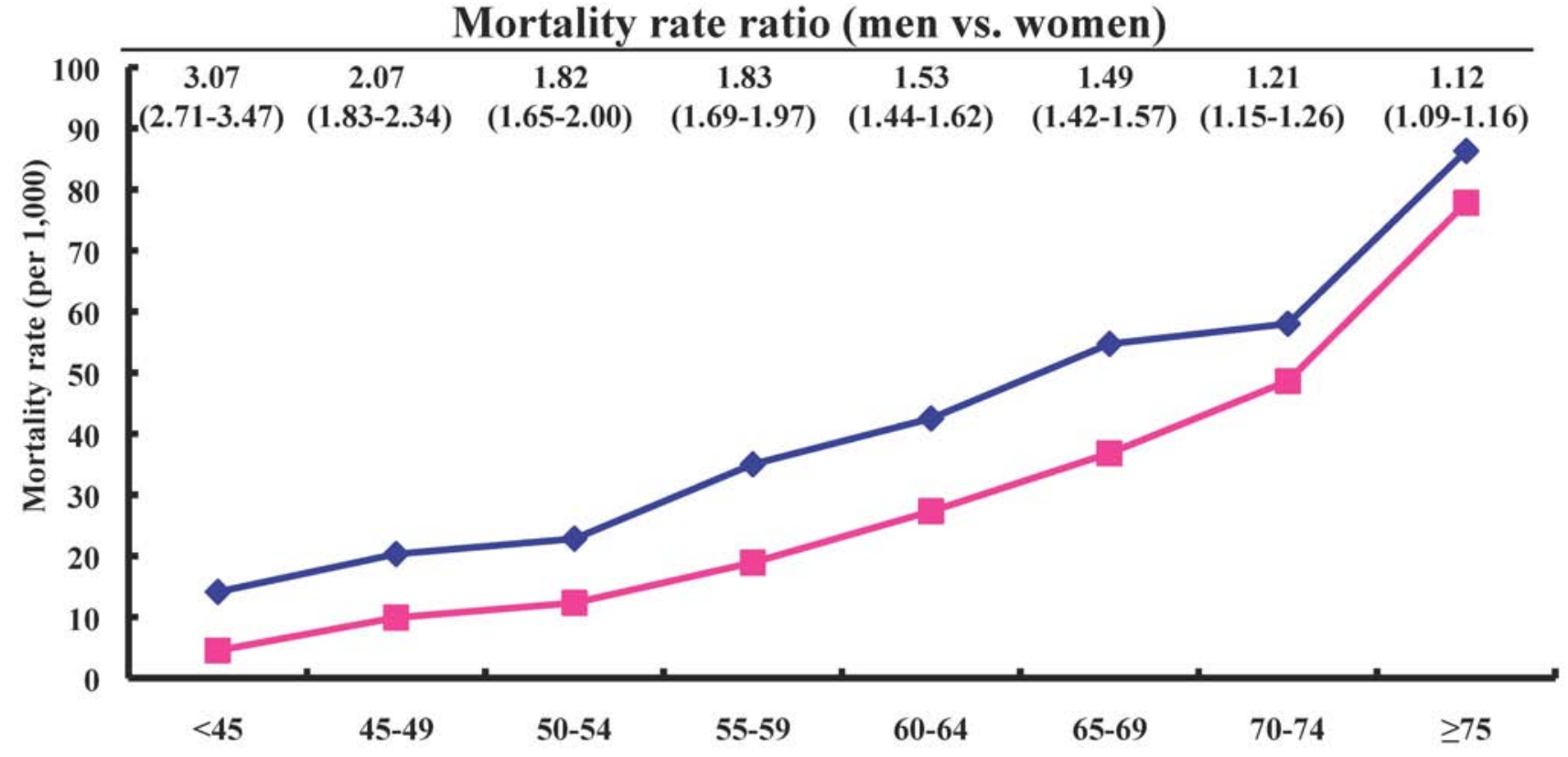

Age (years)

(B)

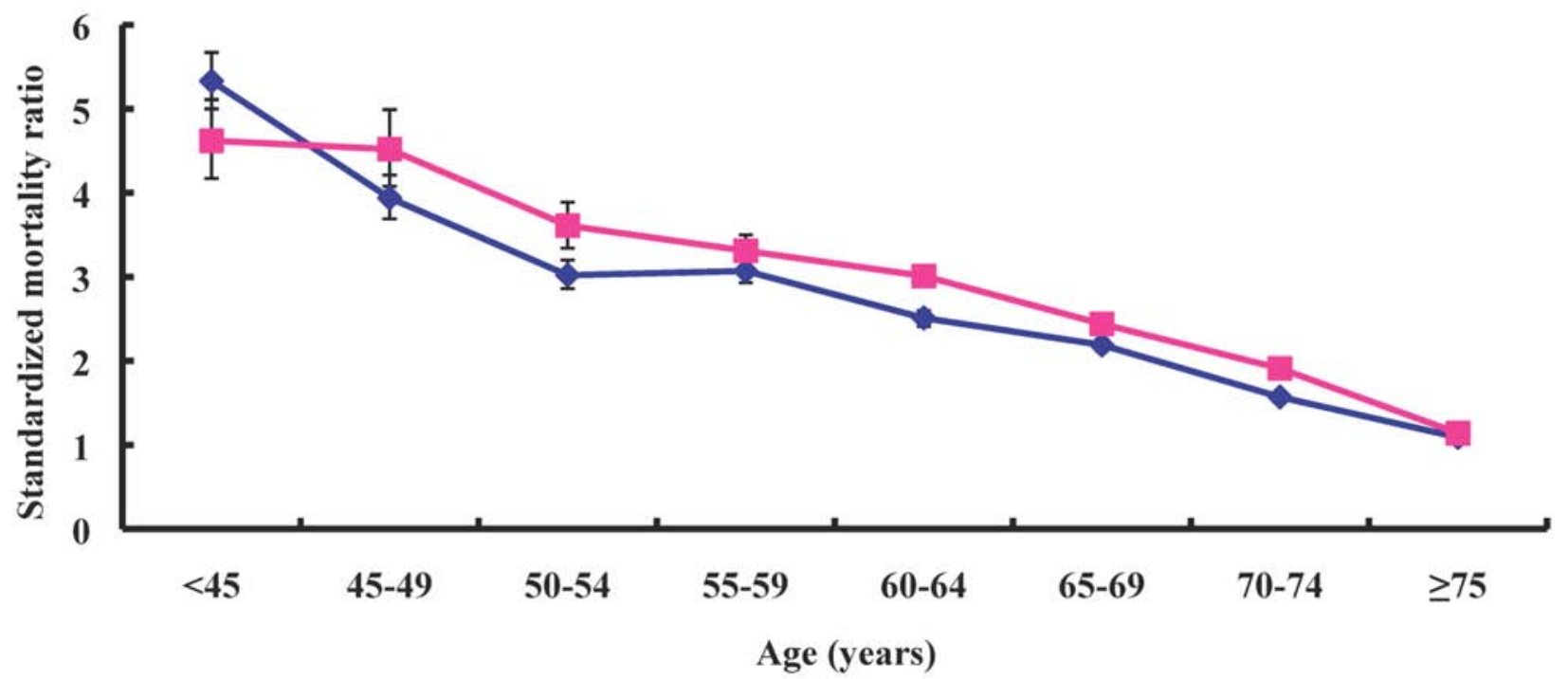

Fig. (3). Age- and sex-specific mortality rates (per 1,000 person-years) (A) and standardized mortality ratios (B) in diabetic patients in Taiwan. Mortality rate ratios for the diabetic men $v s$ the diabetic women in different age groups are shown on top of Fig. (3A). The vertical bars in Fig. (3B) indicate 95\% confidence intervals (figures adapted from reference No. 6; diamond: men, square: women).

Analyzing the prevalence of lower extremity amputation (cohort II), stroke (cohort III) and coronary heart disease (unpublished data in cohort III) in the diabetic patients suggests significant age and sex differences in macrovascular complications with male preponderance. The analysis of incidence of hypertension in cohort II also revealed a higher risk in the diabetic men than the diabetic women [10]. Because mortality in the diabetic patients is closely associated with the macrovascular complications, for which hypertension is the most important risk factor, the higher risk of hypertension and the higher prevalence of macrovascular complications in the diabetic men than women in our population gives good explanation for the higher mortality in the diabetic men.

Obesity probably explains the increase of incidence of diabetes in recent years in Taiwan. Further in-depth analyses of the obesity and parental diabetes accompanying the incident cases during 1992-1996 in cohort II showed that the proportion of obesity in the incident cases increased with decreasing age while the parental history of diabetes decreased dramatically from 1992 to 1996 in the youngest incident cases of <35 years old (Fig. 5). The reason for the 
(A)

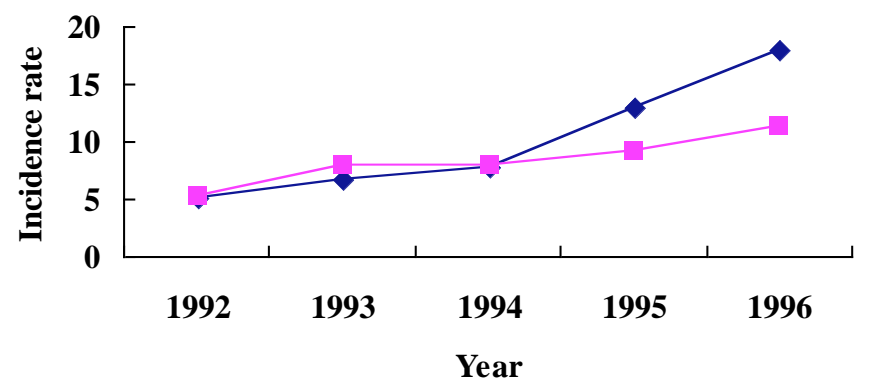

(B)

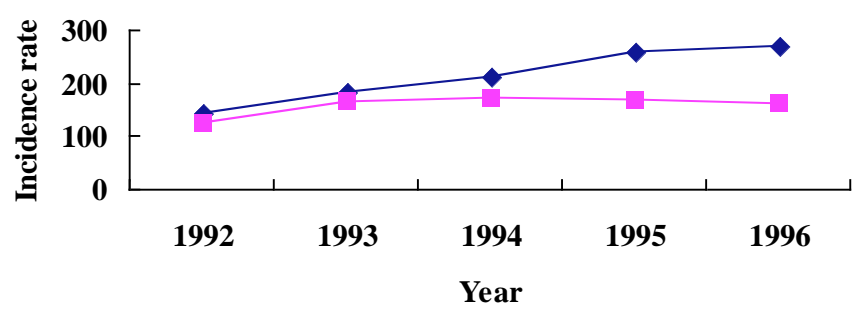

(C)

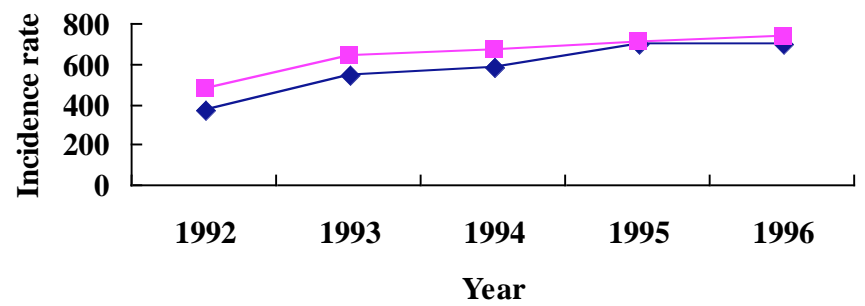

(D)

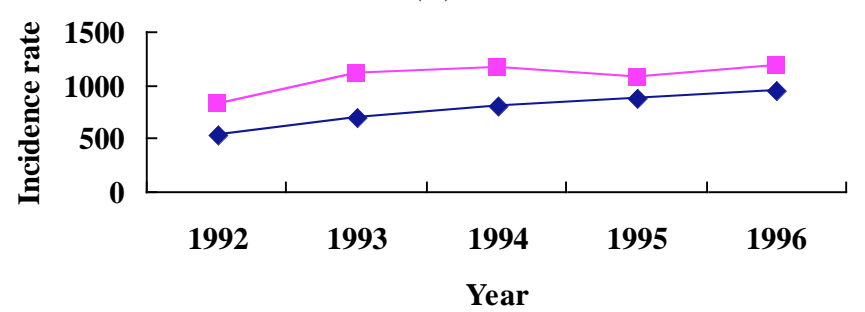

(E)

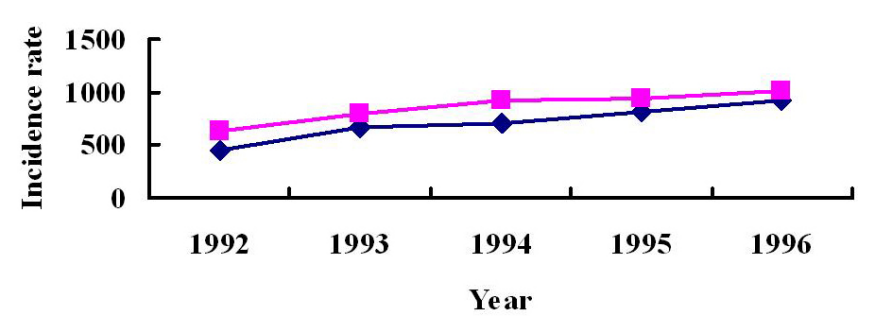

Fig. (4). Age-specific incidence (per 100,000) of diabetes in Taiwan during the period of 1992-1996 for men (diamond) and women (square). (A) age < 35 years, (B) age 35-44 years, (C) age 45-54 years, (D) age 55-64 years and $(\mathbf{E})$ : age $\geq 65$ years (figures adapted from reference No. [7]).

reversal of sex preponderance in diabetes incidence is probably linked to the higher prevalence of obesity in the male population in the young generation [15-17]. In three surveys among school children of 12-15 years old in 1980-1982, 1986-1988 and 1994-1996, the respective prevalence of obe- sity was $12.4 \%, 14.8 \%$ and $16.4 \%$ for boys; and $10.1 \%$, $11.1 \%$ and $11.1 \%$ for girls [15]. While comparing the data obtained in the second [16] and the third [17] National Nutrition and Health Survey in 1986-1988 and 1993-1996, respectively, body mass index has increased in all ages from 4 to $\geq 65$ years in either sex. It was also interesting that obesity was more prevalent in men in the younger ages, but more prevalent in women in the older ages [14]. This partly explained the incidence rates of diabetes being higher in males in the younger ages and higher in females in the older ages.

(A)

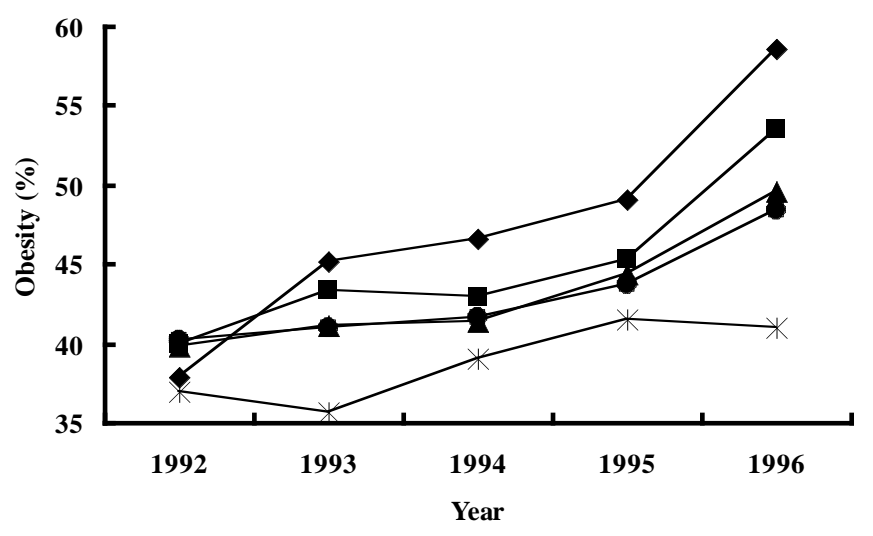

(B)

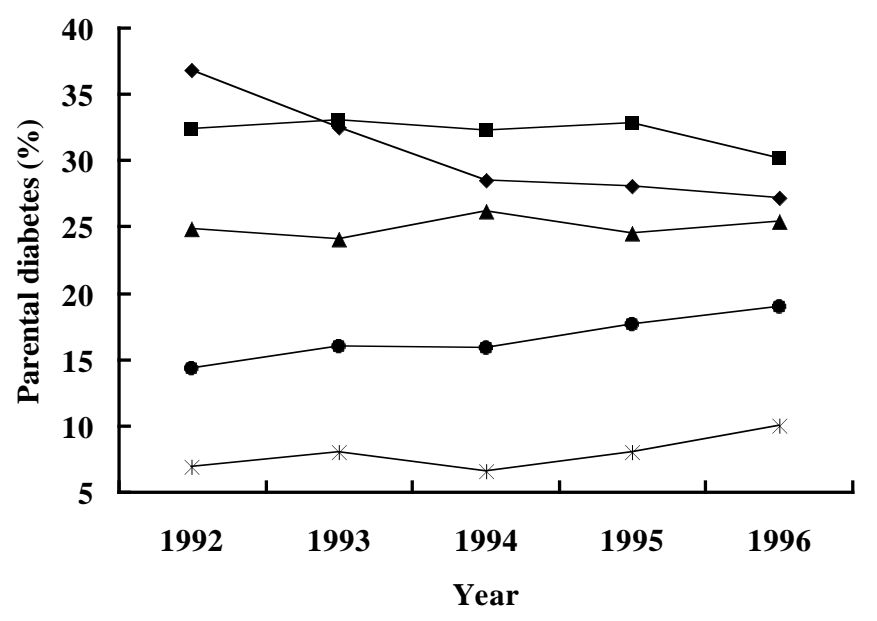

Fig. (5). The trends of obesity (defined by body mass index $\geq 25$ $\mathrm{kg} / \mathrm{m}^{2}, \mathbf{A}$ ) and parental diabetes (B) in the incident cases of type 2 diabetes mellitus with regards to onset age during 1992-1996 in Taiwan. Onset age is divided into $<35$ (diamond), 35-44 (square), 45-54 (triangle), 55-64 (sphere) and $\geq 65$ years (star).

The NHI is a very unique health care system in Taiwan. The establishment of a large diabetes cohort from this system seems to be appropriate for monitoring the incidence trend by using the data periodically. Prospective follow-up of the cohort not only gives valuable information on the epidemiologic transition of diabetes, but also provides new important observations on the health of the diabetic patients. For example, by following the survival of our established cohort of diabetic patients and comparing their mortality causes to those of the general population of Taiwan, we recently observed a significantly higher risk of mortality from breast cancer in the diabetic women [18] and from bladder cancer in diabetic patients of either sex [19]. Epidemiologic data are still lacking to answer whether the medical care to- 
day has greatly improved the survival of diabetic patients when they experience a major clinical event. By periodically comparing the mortality of diabetic patients diagnosed at different time frame, we will be able to answer this question in our future study.

\section{CONCLUSIONS}

A close observation of the epidemiologic transition is mandatory for taking timely and appropriate measures for the prevention and treatment of diabetes. Increasing prevalence of diabetes with female preponderance had been observed for decades in Taiwan, which can be explained partly by the higher risk of morbidity and mortality in the diabetic men and an overall higher incidence in women of all ages. The increasing trend in diabetes incidence can be explained by an increasing prevalence of obesity in the general population, which is especially remarkable in the younger generation of the male sex. Therefore the sex preponderance in diabetes is expected to change from women to men in Taiwan rolling from the past decades to the future. Obesity seems to be a modifiable risk factor for effective intervention to curb the trend.

\section{ACKNOWLEDGEMENTS}

The author thanks the following institutes for their continuous support on the epidemiologic studies of diabetes mellitus and arsenic-related health hazards: the New Century Health Care Promotion Foundation; the Department of Health (DOH89-TD-1035; DOH97-TD-D-113-97009), the National Taiwan University Hospital Yun-Lin Branch (NTUHYL96.G001) and the National Science Council (NSC-86-2314-B-002-326, NSC-87-2314-B-002-245, NSC88-2621-B-002-030, NSC89-2320-B002-125, NSC-902320-B-002-197, NSC-92-2320-B-002-156, NSC-93-2320B-002-071, NSC-94-2314-B-002-142, NSC-95-2314-B-002311 and NSC-96-2314-B-002-061-MY2), Taiwan.

\section{REFERENCES}

[1] King H, Aubert RE, Herman WH. Global burden of diabetes, 19952025: prevalence, numerical estimates, and projections. Diabetes Care 1998; 21: 1414-31

[2] Wild S, Roglic G, Green A, Sicree R, King H. Global prevalence of diabetes: estimates for the year 2000 and projections for 2030 . Diabetes Care 2004; 27: 1047-53.
[3] Roglic G, Unwin N, Bennett PH, et al. The burden of mortality attributable to diabetes: realistic estimates for the year 2000 Diabetes Care 2005; 28: 2130-5.

[4] Tai TY, Yang CL, Chang CJ, et al. Epidemiology of diabetes mellitus among adults in Taiwan, R.O.C. J Med Assoc Thai 1987; 70(Suppl 2): 42-8.

[5] Pan WH, Yeh WT, Chang HY, Hwu CM, Ho LT. Prevalence and awareness of diabetes and mean fasting glucose by age, sex, and region: results from the Nutrition and Health Survey in Taiwan, 1993-1996. Diabet Med 2003; 20: 182-5.

[6] Tseng CH. Mortality and causes of death in a national sample of diabetic patients in Taiwan. Diabetes Care 2004; 27: 1605-9.

[7] Tseng CH, Tseng CP, Chong CK, et al. Increasing incidence of diagnosed type 2 diabetes in Taiwan: analysis of data from a national cohort. Diabetologia 2006; 49: 1755-60.

[8] Tseng CH. Prevalence of lower-extremity amputation among patients with diabetes mellitus: Is height a factor? CMAJ 2006; 174 : 319-23.

[9] Tseng $\mathrm{CH}$. Higher risk of hypertension in indigenous type 2 diabetic patients in Taiwan. J Hypertens 2006; 24: 1817-21.

[10] Tseng $\mathrm{CH}$. Exogenous insulin use and hypertension in adult patients with type 2 diabetes mellitus. Arch Intern Med 2006; 166 : 1184-9.

[11] Tseng CH. Effect of parental hypertension and/or parental diabetes on hypertension in Taiwanese diabetic patients. Eur J Clin Invest 2007; 37: 870-7.

[12] Tseng CH. Body mass index and blood pressure in adult type 2 diabetic patients in Taiwan. Circ J 2007; 71: 1749-1754.

[13] Tseng CH. Betel nut chewing is associated with hypertension in Taiwanese type 2 diabetic patients. Hypertens Res 2008; 31: 41723.

[14] Tseng CH, Chong CK, Sheu JJ, Wu TH, Tseng CP. Prevalence and risk factors for stroke in type 2 diabetic patients in Taiwan: A cross-sectional survey of a national sample by telephone interview. Diabet Med 2005; 22: 477-82.

[15] Chu NF. Prevalence of obesity in Taiwan. Obes Rev 2005; 6: 2714.

[16] Kao MD, Huang MS, Tzeng NY, Lee NY, Shieh MJ. The nutritional status in Taiwan: anthropometric measurement, 1986-1988. I. Body weight and body height (in Chinese). J Chin Nutr Soc 1991; 16: 63-84.

[17] Department of Health. National Nutrition and Health Survey in Taiwan, 1993-1996 (in Chinese). December 1998.

[18] Tseng CH, Chong CK, Tai TY. Secular trend for mortality from breast cancer and the association between diabetes and breast cancer in Taiwan between 1995 and 2006. Diabetologia 2009; 52: 2406.

[19] Tseng CH, Chong CK, Tseng CP, Chan TT. Age-related risk of mortality from bladder cancer in diabetic patients: A 12-year follow-up of a national cohort in Taiwan. Ann Med (in press). 